\title{
ADHERENCIA DE Candida albicans A RESINAS ACRÍLICAS Y POLIAMIDAS. ESTUDIO IN VITRO
}

Sonia Elena Pineda-Higuita ${ }^{1}$

Josefina Mosquera-Palomino²

\section{RESUMEN}

Objetivo: Determinar la adherencia de Candida albicans a resinas acrílicas y poliamidas. Materiales y métodos: Estudio experimental in vitro en el cual se realizaron 12 prótesis dentales, seis parciales acrílicas, elaboradas bajo la técnica de termo-curado, y seis en poliamidas mediante inyección; para el análisis se tomaron muestras de $2 \mathrm{~mm}$ de bordes irregulares, de diferentes zonas de la prótesis y se evaluaron variables tales como temperatura, tiempo de cocción, tipo de pulido, tipo de material para brillar, presencia de porosidades, tipo de abrasivo y aditamento para retirar excesos; las muestras se mantuvieron en agua destilada por dos días para retirar los excesos de monómero y posteriormente fueron incubadas en una suspensión de Candida albicans de referencia ATCC 10231 y evaluadas por microscopía electrónica de barrido. Resultados: En las resinas elaboradas en acrílicos se observaron poros, grietas, blastonidias y pseudomicelios. En las muestras de poliamidas también se observaron estos mismos hallazgos, pero en menor proporción. Conclusión: Se encontró adherencia en las 12 muestras evaluadas, se halló asociación estadísticamente significativa entre tipo de pulido y tipo de abrasivo con un valor de $(\mathrm{p}<0,05)$.

Palabras clave: Candida albicans, prótesis dentales, adherencia.

\section{ADHERENCE OF Candida albicans TO ACRYLIC RESINES AND POLYAMIDES. STUDY IN VITRO}

\section{ABSTRACT}

Objective: To determine the adherence of Candida albicans to polyamide and acrylic resins. Materials and Methods: In this experimental in vitro study 12 dental prostheses were made, 6 acrylic partial dentures which were elaborated under the heat-curing technique and 6 prosthesis made of polyamide by injection; $2 \mathrm{~mm}$ samples with irregular borders were taken from different areas of the prosthesis for analysis and variables such as temperature, heating time, polishing time, material used to shine, presence of porosities, accessory and abrasive used to remove excess were evaluated; the samples were held in distilled water for two days to remove the monomer excess and then they

\footnotetext{
${ }^{1}$ Magíster en Salud Pública Universidad CES. Profesora del Programa Laboratorio de Prótesis Dental e Investigadora Grupo de Investigación en Salud y Comunidad de la Fundación Universitaria Autónoma de las Américas. Medellín, Colombia. Autor para correspondencia. E-mail: sonia.pineda@uam.edu.co

${ }^{2}$ Aspirante a Magíster en Ingeniería Ambiental Universidad Pontificia Bolivariana. Profesora del Programa Laboratorio de Prótesis Dental e Investigadora Grupo de Investigación en Salud y Comunidad de la Fundación Universitaria Autónoma de las Américas. Medellín, Colombia. Correo electrónico: josefina.mosquera@uam.edu.co
} 
were incubated in a Candida albicans suspension strain ATCC 10231 and evaluated by scanning electron microscopy (SEM). Results: Porosities, cracks, blastospore and pseudohyphae were observed on the resins made from acrylic. The same results were observed on the samples made from polyamide to a lesser extent. Conclusion:

\section{INTRODUCCIÓN}

La salud oral en general se considera deficiente entre las personas adultas, debido a la elevada prevalencia de edentulismo, caries y enfermedad periodontal. Para rehabilitar al paciente del edentulismo, generalmente se confeccionan prótesis removibles (PR). El uso de estas altera las condiciones orales, causando queratinización y lesiones en la mucosa, cuya etiología puede ser infecciosa, mecánica o alérgica. Otros factores tales como la higiene deficiente, las irregularidades superficiales los desajustes y la presión negativa en la zona de contacto prótesismucosa favorecen la formación del biofilm, permitiendo la acción de microorganismos (1).

La lesión de la mucosa oral más prevalente en portadores de PR es la estomatitis prótesis (ESP), proceso inflamatorio que afecta la mucosa. El principal factor etiológico de ESP es una levadura que pertenece al reino fungi, el cual incluye eucariotas del tipo de los hongos unicelulares o levaduriformes y pluricelulares o filamentosos

En la actualidad, el reino fungi se divide en dos subreinos, Dykaria, el cual agrupa las divisiones Ascomycota y Basidiomycota, y el llamado Hongos Basales, que agrupa al resto de los hongos. Dentro del segundo subreino se ubican aquellas especies que antes pertenecían a la división Zygomycota (zigomicetes) y que, se ha demostrado, constituyen un grupo polifilético, por lo que dicha categoría ha sido eliminada en las nuevas clasificaciones
Adhesion was found on the 12 samples evaluated. A statistically significant association was found between polishing type and abrasive type with a value of $(\mathrm{p}<0.05)$.

Key words: Candida albicans, dental prostheses, adherence.

A la división Ascomycota pertenecen la mayoría de hongos patógenos, tanto los levaduriformes como los filamentosos. Entre los primeros es de destacar en los últimos años el incremento de las infecciones por Candida (2).

Las especies de la Candida son hongos que se encuentran con frecuencia en la boca de individuos sanos y como tales se pueden considerar residentes normales de la microflora oral, es decir existen sin causar daño en las membranas mucosas, como las orejas, ojos, tracto gastrointestinal, nariz, órganos reproductores, senos paranasales, la piel, las heces, vagina, cavidad oral, etc; se le conoce como flora normal. Cuando ocurre un desequilibrio que causa un crecimiento excesivo se convierte en una micosis llamada candidiasis (3-5)

Dentro de las diferentes especies de Candida se encuentra Candida albicans, considerada una especie productora de infecciones en la cavidad oral. La literatura reporta que alrededor de $30 \%$ al $50 \%$ de las personas llevan consigo este organismo y la tasa aumenta con la edad y en personas que portan prótesis dentales. Existen otras especies como, C. glabrata, C. guillermondii, C. krusei, C. parapsilosis, C. pseudotropicalis, C. stellatoidea, C. tropicalis, que también hacen parte de mucosa oral $(6,7)$.

Varias especies de microorganismos coexisten en un ciclo dinámico biológico en la cavidad oral. La mayoría de estos microorganismos han sido implicados en procesos patológicos, y de adhesión a superficies como la mucosa oral y materiales dentales. C. albicans ha sido estudiado 
en dichos procesos de adhesión e importante en la patogénesis de estomatitis protésica. Además, esta adherencia se ha visto influenciada por otros factores, tales como la rugosidad de la superficie, película salival, la energía libre de la superficie y el tipo de material de la prótesis (8-10).

Candida y Streptococcus mutans han sido microorganismos aislados de prótesis dentales e incluso en ausencia de estomatitis protésica (11).

Diferentes errores en el procesado y manipulación de la masa del polímero en las bases de prótesis dentales como son: vaporización del monómero por exceso de temperatura, falta de homogeneidad en el momento de la polimerización y presión inadecuada, dan lugar a la aparición de poros, los cuales se convierten en lugar apto para el crecimiento de diferentes tipos de microorganismos, entre ellos C. albicans.

Por todo lo expuesto anteriormente, el objetivo de esta investigación fue determinar la adherencia de Candida albicans a resinas acrílicas y poliamidas.

\section{MATERIALES Y MÉTODOS}

Estudio experimental in vitro en el cual se realizaron 12 prótesis dentales prótesis, seis parciales acrílicas elaboradas bajo la técnica de termo-curado y seis en poliamidas elaboradas mediante inyección; para el análisis se tomaron muestras de $2 \mathrm{~mm}$ de bordes irregulares, de diferentes zonas de la prótesis y se evaluaron variables tales como temperatura, tiempo de cocción, tipo de pulido, tipo de material para brillar, presencia de porosidades, tipo de abrasivo y aditamento para retirar excesos, las muestras se mantuvieron en agua destilada por dos días para retirar los excesos de monómero que pudieran quedar después de la polimerización; posteriormente se desinfectaron con alcohol al $70 \%$ durante tres minutos y luego fueron lavadas con agua destilada. Una vez realizados estos procedimientos se incubaron en una suspensión de Candida albicans de referencia
ATCC 10231 y evaluadas por microscopía electrónica de barrido. Este proyecto fue avalado por convocatoria interna de la Fundación Universitaria Autónoma de las Américas, Código P36-2014.

\section{Cultivo del microorganismo}

Se utilizó una cepa de referencia ATCC 10231 de la colección de microorganismos de KWIKSTIK ${ }^{\mathrm{TM}}$ PLUS producido por Microbiologics INC de la cual se realizó la siembra en medio Agar Sabouraud Dextrosa; una vez triturado el material que contenía el liofilizado, se incubó durante 48 horas a $37^{\circ} \mathrm{C}$ con el fin de que las levaduras se desarrollaran completamente. Después de este período de tiempo se obtuvo crecimiento de unas colonias lisas y de color blanco compatibles con C. albicans, las cuales fueron corroboradas con la prueba tubo germinal para esta prueba se inoculó una gota de albumina de huevo en una lámina portaobjeto estéril y una pequeña porción de la cepa a identificar, y se mezcló con la ayuda de un asa bacteriológica. Esta preparación se cubrió con una lámina portaobjeto, se dejó por espacio de 2 horas a $37^{\circ} \mathrm{C}$ y luego se observó al microscopio donde se evidenció la formación del tubo germinal.

\section{Preparación de la muestra y ensayo de adherencia}

Del cultivo obtenido se tomaron cuatros asadas microbiológica y se resuspendieron en $8 \mathrm{~mL}$ de BHI (infusión cerebro corazón) medio líquido utilizado para cultivar microorganismos. Esta solución se mantuvo en una incubadora orbital a $37^{\circ} \mathrm{C}$ con agitación de $(40-60 \mathrm{rpm})$ durante 48 horas. Luego se tomó esta solución y se centrifugó 10 minutos a 1,000 rpm, posteriormente se hicieron dos lavados con PBS (solución buffer fosfato) para remover las células que no lograron adherirse a las prótesis y se fijaron en formaldehido al $4 \%$, la desecación final se realizó por secado de punto crítico. Las muestras se recubrieron con oro, posteriormente 
se realizó la lectura en microscopio electrónico de barrido y se obtuvieron fotografías con aumentos de 500x, 1000x, 2000x, 3000x y 5000x, las cuales se utilizaron para observar la adherencia del hongo.

\section{RESULTADOS}

Se analizó la adherencia tanto de resinas acrílicas como a poliamidas mediante microscopia electrónica de barrido. La resina acrílica se elaboró bajo la técnica de termo-curado y la de las poliamidas mediante inyección, para el análisis se tomaron muestras de $2 \mathrm{~mm}$ de borde irregulares, de diferentes zonas de la prótesis. En la figura 1 a se observan blastoconidias con escasos micelios, sobre una superficie lisa, en la figura $1 \mathrm{~b}$ se observan algunas líneas y blastoconidias, en la figura 1 c se observan blastoconidias, pseudomicelios y una microestructura porosa de superficie irregular. En la figura 1d se observan acúmulos de blastoconidas y grandes grietas, una superficie irregular, en la figura 1e se observa una grieta de gran tamaño y escasos blastoconidas y en la figura 1f se observa una excavación de la superficie irregular, escasas blastoconidias y puntos de perforación ( figura 1).

Figura 1. Diferencias en la adherencia de Candida albicans a prótesis dentales elaboradas en resinas acrílicas a las 48 horas de incubación. MEB (microscopio electrónico de barrido) 500x, 1.000x, 2.000x, $3.000 x$ y $5.000 x$.

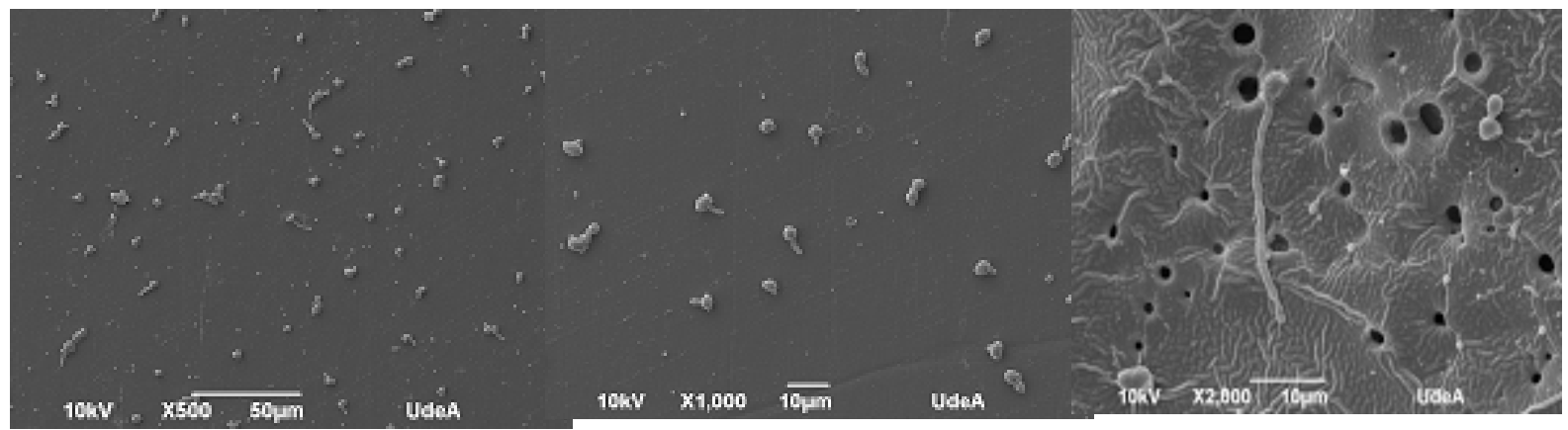

Figura $1 a$

Figura $1 b$

Figura 1c

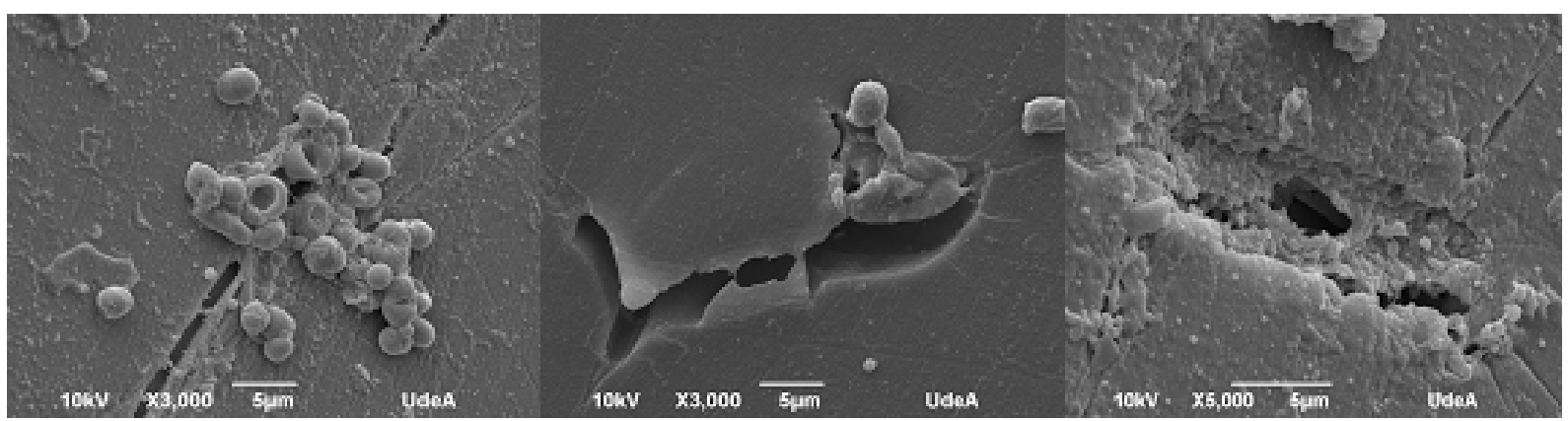

Figura $1 d$

Figura $1 e$

Figura $1 f$ 
Las prótesis dentales elaboradas en poliamidas se trabajaron con una temperatura de $280^{\circ} \mathrm{C}$. Al observar la adherencia por microscopía electrónica de barrido se halló menos crecimiento en relación con las muestras elaboradas en acrílico. En la figura 2a se observan abundantes micelios sobre una superficie lisa con unas algunas líneas, en la figura $2 b$, se observó escaso crecimiento de $C$. albicans al igual que grandes grietas sobre la estructura, muy similar a la figura 2c, sólo que las grietas son mayores; en la $2 \mathrm{~d}$, se observan una excavación en la superficie, abundantes micelios e hifas; en la 2e se observan escasas blastoconidias sobre una superficie irregulary en $2 f$ se observa una excavación profunda, varias capas superpuestas y escasas blastoconidias (figura 2).

Figura 2. Diferencias en la adherencia de Candida albicans a prótesis dentales elaboradas en poliamidas a las 48 horas de incubación. MEB (microscopio electrónico de barrido) 500x, 1.000x y 5.000x.

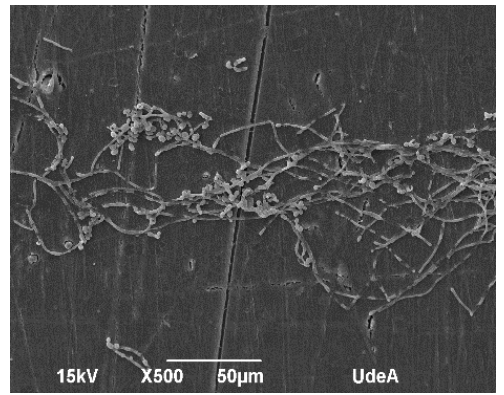

Figura 2 a

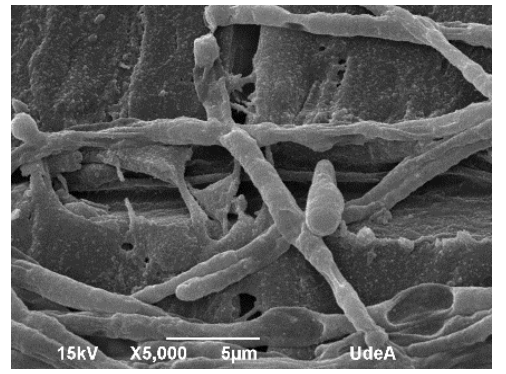

Figura $2 d$

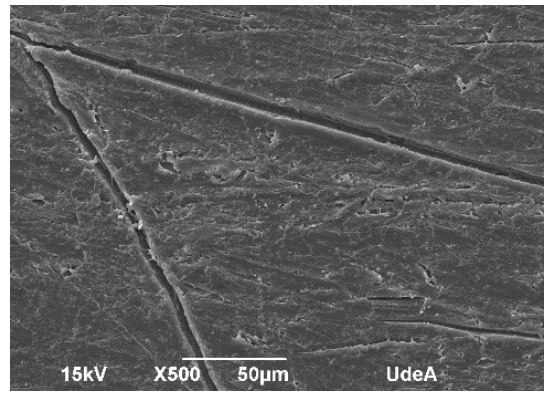

Figura $2 b$

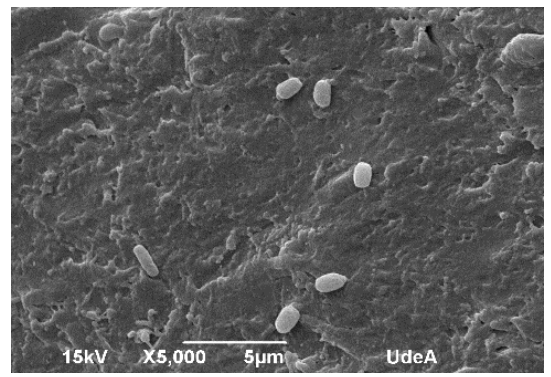

Figura $2 e$

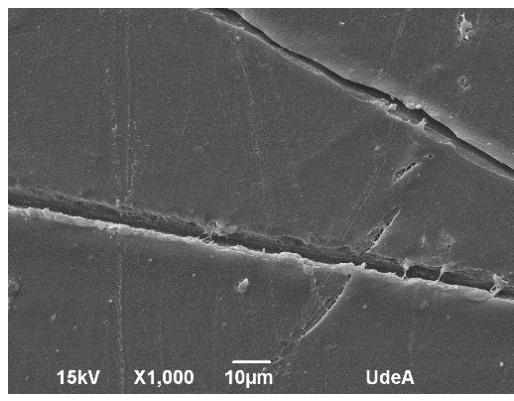

Figura $2 \mathrm{c}$

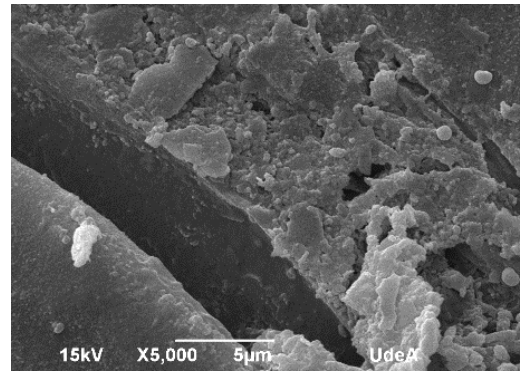

Figura $2 f$
No se encontró diferencia estadísticamente significativa en la adherencia y las variables analizadas tipo de abrasivo, tipo de pulido, temperatura de cocción, presencia de porosidades, tipo de prótesis y material para brillar. Solo se encontró asociación estadística significativa entre tipo de pulido y tipo de abrasivo $(p<0,05)$. La adherencia se había planteado como variable dependiente, pero en el análisis se observó adherencia de C. albicans tanto en resinas acrílicas como en poliamidas siendo menor en esta última (tabla 1). 
Tabla 1. Asociación ente variables independientes.

\begin{tabular}{ccc}
\hline Variables asociadas & Valor chi ${ }^{2}$ & Valor de $p$ \\
\hline Abrasivo y tipo de pulido & 29,481 &, 043 \\
Porosidades y temperatura de cocción &, 800 &, 371 \\
Tipo de pulido y tipo de prótesis & 5,206 &, 267 \\
Tipo de abrasivo y presencia de porosidades & 8,400 &, 299 \\
Tipo de muestra y presencia de porosidades &, 000 & 1,000 \\
Material para brillar y tipo de pulido & 5,321 &, 503 \\
Tipo de cono de silicona para pulir y tipo de pulido & 6,874 &, 550 \\
\hline
\end{tabular}

\section{DISCUSIÓN}

La capa más externa de la pared celular de Candida albicans consta de un complejo de polisacáridos principalmente Manán, Glucán y Quitina, además de proteínas y una proporción variable de lípidos, los cuales están involucrados en la adhesión a las células del huésped, así como a las propiedades antigénicas (12).

En un estudio de adherencia de C. albicans al PMMA realizado por Chandra et al., se observó que este microorganismo a partir de las $11 \mathrm{~h}$ de inoculación, empieza a aglutinarse en las áreas irregulares de la resina acrílica, lo cual crea reservorios protegidos para el hongo. En nuestro estudio se observaron resultados similares, en el caso de las muestras elaboradas en poliamidas la adherencia fue menor (13).

En un estudio realizado por Izumida et al., sobre evaluación in vitro de adherencia de C. albicans a resinas acrílicas modificadas encontraron que la saliva y la rugosidad no promovieron la adhesión de microorganismos y esta disminución en la adhesión la asociaron con la presencia de revestimientos, en nuestro estudio la resina acrílica no tuvo ninguna modificación y la adherencia de C. albicans fue evidente (14)

El pulido y acabado de las prótesis dentales influyen en la adherencia de microorganismos y hongos como la C. albicans (15)
Estudios realizados demuestran que C. albicans se adhiere más en superficies rugosas de las resinas acrílicas que en las lisas, lo cual se corrobora en nuestra investigación (16)

Se están desarrollando métodos que permitan reducir la adherencia de C. albicans en las resinas acrílicas polimerizadas térmicamente como es la aplicación de plasma frío a presión atmosférica a la superficie de la resina. El plasma frío parecía ser una estrategia prometedora y conveniente para evitar la prematura adherencia de $C$. albicans en resinas acrílicas, esto beneficiaría en gran medida las aplicaciones dentales (17)

En un estudio realizado por Koch et al. se encontró asociación estadísticamente significativa en la proliferación de C. albicans según el material que se utiliza para la fabricación de bases de la dentadura ( $p<0,001)$. La adherencia del hongo no depende tanto de la polimerización de la resina. Para el caso nuestro no se obtuvo este tipo de asociación (18)

Sobre las limitaciones, inicialmente estaba programado el análisis con 20 muestras y solo se pudo realizar el ensayo de adherencia en 12 muestras, lo cual pudo haber interferido con la no obtención de diferencias estadísticamente significativas, en este caso se tendría que aumentar el tamaño de la muestra. El análisis por microscopía electrónica de barrido es una técnica apropiada para valorar la adherencia de microorganismos a diferentes tipos de superficies, así como sus características. 


\section{CONCLUSIONES}

Los resultados del presente estudio corroboran la capacidad de adherencia de Candida albicans a resinas acrílicas y poliamidas; los procedimientos de elaboración y acabado final son un factor determinante en la adherencia de este microorganismo a materiales dentales.

La adherencia a poliamidas fue inferior que a las resinas acrílicas, sin embargo, las áreas donde hay porosidades, rugosidades y mal pulido fue donde hubo mayor crecimiento, ya que estos sitios pueden proporcionar una mayor retención para las células.

C. albicans tiene gran capacidad de adherencia a materiales no solo a células del hospedero sino también a materiales inertes en este caso a poliamidas y resinas acrílicas, esto en gran parte se debe a lo que reporta la literatura sobre las características químicas y estructurales de su pared celular.

El análisis por microscopía electrónica de barrido es una técnica apropiada para valorar la adherencia de microorganismos a diferentes tipos de superficies, así como sus características.

\section{AGRADECIMIENTOS}

A los estudiantes Cindy Yadine Mejía, María Alejandra Correales Carmona, Jonathan Gaviria Franco y Dawinson Restrepo, estudiantes del Programa Académico de Laboratorio de Prótesis Dental de la Fundación Universitaria Autónoma de las Américas por la elaboración de las estructuras y al docente Carlos Ignacio Vélez por el acompañamiento a los estudiantes. Al Bacteriólogo Camilo Beltrán por su apoyo y al Laboratorio de Materiales de la Universidad de Antioquia, donde se realizó el análisis por microscopio electrónico de barrido. A la profesional Lorena Sánchez Diosa por sus servicios de apoyo para el análisis estadístico.

\section{FINANCIACIÓN}

Este trabajo fue financiado por convocatoria del Departamento de Investigación de la Fundación Universitaria Autónoma de las Américas Proyecto Código P36-2014.

\section{CONFLICTO DE INTERESES}

Los autores declaran no tener ningún conflicto de interés relacionado con este artículo. 


\section{REFERENCIAS}

1. Lee Muñoz $X$ et al. Ocurrencia de levaduras del género Candida y estomatitis protésica antes y después del tratamiento rehabilitador basado en prótesis removible. Rev Clin Periodoncia Implantol Rehabil Oral. 2015; 8(1):31-37.

2. Guarro J. Taxonomía y biología de los hongos causantes de infección en humanos. Enferm Infecc Microbiol Clin. 2012; 30(1):33-39.

3. , Marsh PD, Martin MV, . Microbiología oral. Quinta edición. Amolca Actualidades Médicas, C.A; 2011.

4. Singh A, Verma R, Murari A, Agrawal A. Oral candidiasis: An overview. J Oral Maxillofac Pathol. 2014 Sep; 18(Suppl 1): S81-S85.

5. Yang $Y L$, Leaw SN, Wang AH, Chen HT, Cheng WT, Lo HJ. Characterization of yeasts colonizing in healthy individuals. Med Mycol 2011; 49:103-6.

6. Terezhalmy GT, Huber MA. Oropharyngeal candidiasis: Etiology, epidemiology, clinical manifestations, diagnosis, and treatment. Crest Oral-B at dentalcare.com Contin Educ Course. 2011:1-16.

7. Prasanna KR. Oral candidiasis - A review. Scholarly J Med. 2012; 2:6-30.

8. Rodríguez Acosta EJ, da Silva PM, Jacobina M, Lara VS, Neppelenbroek KH, Porto VC Candida albicans adherence to denture base material: chemical disinfection and the effect of acquired salivary pellicle formation. Journal of Prosthodontics 2015; 24:200-206.

9. Akalın-Evren B, Kulak-Özkan Y, Ozcan M, Kadir T. Candida albicans adhesion on reinforced polymethylmethacrylate denture resin: effect of fibre architecture and exposure to saliva. Gerodontology. 2014; 31(3):194-201.

10. Pan H, Wang G, Pan J, Ye G, Sun K, Zhang J, Wang J. Cold plasma-induced surface modification of heat-polymerized acrylic resin and prevention of early adherence of Candida albicans. Dental Materials Journal 2015; 34(4): 529-536.

11. Al-Bakri IA, Harty D, Al-Omari WM, Swain MV, Chrzanowski W, Ellakwa A. Surface characteristics and microbial adherence ability of modified polymethylmethacrylate by fluoridatedglass fillers. Aust Dent J. $2014 ; 59(4): 482-9$.

12. Sato $M$, Ohshima $T$, Maeda $N$, Ohkubo C. Inhibitory effect of coated mannan against the adhesion of Candida biofilms to denture base resin. Dental Materials Journal 2013; 32(3): 355-360.

13. Chandra J. Biofilm formation by the fungal pathogen Candida albicans: Development, architecture, and drug resistance. Journal of Bacteriology 2001; 183(18): 5385-5394.

14. Izumida FE, Moffa EB, Vergani CE, Machado AL, Jorge JH, Giampaolo ET. In vitro evaluation of adherence of Candida albicans, Candida glabrata, and Streptococcus mutans to anacrylic resin modified by experimental coatings. Biofouling. 2014; 30(5):525-33.

15. Tsarev VN, Ippolitov EV, Trefilov AG, Arutiunov SD, Pivovarov AA. Features of adhesion of anaerobic periodontopathogenic bacteria and Candida albicans fungi to experimental samples of basis dental plastic depending on surface roughness and polishing method. Zh Mikrobiol Epidemiol Immunobiol. 2014; (6):21-7.

16. Mayahara M, Kataoka R, Arimoto T, Tamaki Y, Yamaguchi N, Watanabe $Y$, Yamasaki Y, Miyazaki T. Effects of surface roughness and dimorphism on the adhesion of Candida albicans to the surface of resins: scanning electron microscope analyses of mode and number of adhesions. J Investig Clin Dent. 2014; 5(4):307-12. doi: 10.1111/jicd.12055.

17. Pan H, Wang G, Pan J, Ye G, Sun K, Zhang J, Wang J.Cold plasma-induced surface modification of heat-polymerized acrylic resin and prevention of early adherence of Candida albicans. Dent Mater J. 2015; 34(4):529-36. doi: 10.4012/dmj.2015-035.

18. Koch C, Bürgers R, Hahnel S. Candida albicans adherence and proliferation on the surface of denture base materials. Gerodontology. 2013; 30(4):309-13. doi: 10.1111/ger.12056. 